\title{
Celecoxib-Induced Bullous Pemphigoid: Report Of The First Case
}

\author{
D Yang, I Brownell, M Duvic
}

\section{Citation}

D Yang, I Brownell, M Duvic. Celecoxib-Induced Bullous Pemphigoid: Report Of The First Case. The Internet Journal of Anesthesiology. 2006 Volume 14 Number 2.

DOI: $\underline{10.5580 / 867}$

\begin{abstract}
A 72-year-old woman developed a bullous eruption affecting her oral mucous membranes, hands, and feet. A skin biopsy revealed subepidermal bullae with lymphocytes. Immunofluorescence showed heavy IgG and C3 deposition at the basement membrane zone. Bullous pemphigoid was diagnosed on the basis of clinical, histopathologic, and immunofluorescence findings. The bullae developed after recent ingestion of celecoxib and subsided soon after discontinuation. We believe this case represents the first reported case of celecoxib-induced bullous pemphigoid.
\end{abstract}

\section{INTRODUCTION}

Bullous pemphigoid (BP) is the most common autoimmune blistering skin disease and occurs with increasing frequency in elderly patients. The cause is usually unknown, but there have been cases attributed to drugs. In drug-induced BP, the clinical, histologic, and immunopathologic features are identical to those in idiopathic disease, but induced by systemic ingestion or local use of certain drugs. $\left[_{1}\right]$ This is the first reported case of celecoxib-induced BP.

\section{CASE REPORT}

A 72-year-old woman with a past medical history significant for recurrent colon cancer was admitted for worsening mucositis and bullous eruption of her toes, fingers, and mucous membranes for two weeks. Approximately two years prior to presentation, she had been diagnosed with colon cancer that was treated with sigmoid resection with negative lymph nodes. She subsequently received the first two courses of 5-fluorouracil and leucovorin, but chemotherapy was discontinued because of severe toxicity including mucositis, fatigue, and dehydration. She continued to receive regular follow-up with no recurrence until about six months prior to presentation, when she began noticing bloody stools. An elevated CEA two months later was followed by a negative colonoscopy. However repeat colonoscopy revealed an obstruction which was deemed unresectable, and capecitabine (1500 mg at night for two weeks) along with celecoxib (200 mg twice a day) was chosen for chemotherapy. She tolerated the first cycle complaining only of gum soreness without mucositis or new skin lesions. After her second cycle, she developed extensive mucositic lesions and erythema of her soft palate, buccal mucosa, and lower lip. About one week later, she developed tense blisters on her toes and fingers as well as a few blisters in her oropharynx. (Figure 1) Her chemotherapy was held in addition to all nonessential medications. With discontinuation of her medications and supportive care, the mucositis and bullous lesions improved.

\section{Figure 1}

Figure 1: Tense bullae on oral mucosa amongst mucositic lesions

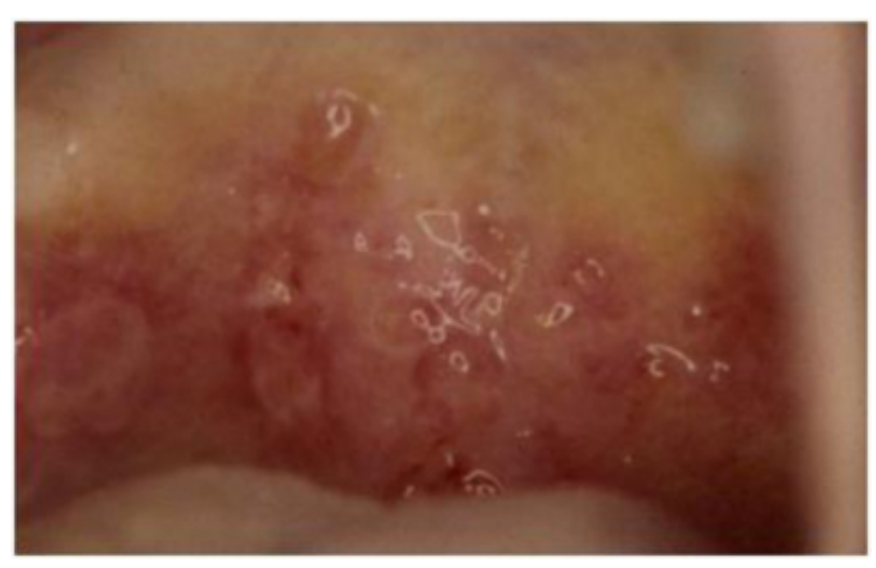

A skin biopsy from left foot was performed which showed a subepidermal blister with lymphocytes. Immunoflourescence revealed a heavy IgG and C3 deposition at the basement 
membrane zone consistent with bullous pemphigoid.

\section{DISCUSSION}

Drug-induced BP presents similarly to idiopathic BP except that it is temporally related to a drug, systemic or local, and it normally clears after discontinuation of the inciting agent. There have been at least 30 systemic drugs described in association with drug-induced BP. [1] (Table 1) Thiol compounds and sulfonamide derivatives have been commonly implicated whereas most other drugs have only occasionally been reported. [2] In most cases, patients have not been rechallenged and thus allowing for the possibility of coincidence. Three patients were rechallenged with phenacetin, furosemide, and tiobutarit (D-penicillamine analogue) respectively with subsequent recurrence of BP. $\left[{ }^{2}\right]$

\section{Figure 2}

Table 1: List of systemic drugs reported to induce bullous pemphigoid $[1,2]$

\begin{tabular}{l|l|}
\hline Actinomycin D & Methyldopa \\
Amozicillin & Nadolo1 \\
Ampicillin & Omeprazole \\
Anti-influenza vaccine & Penicillamine \\
Arsenic & Penicillin \\
Azapropazone & Phenacetin \\
Captopril & Placental extracts \\
Chloroquine & Potassium iodide \\
Clonidine & Practolol \\
Dactinomycin & Psoralens with UVA \\
Enalapril & Risperidone \\
Flupenthisol & Salicylazosulfapyridine \\
Furosemide & Sulfonamide \\
Gold thyosulfate & Tetanus tosoid \\
Tbuprofen & Thiopronin \\
Interleukin-2 & Tiobutarit \\
Mefenamic acid & Tolbutamide \\
&
\end{tabular}

Although it is difficult to disprove coincidence as the cause of BP, some clinical factors point to a drug origin. Often patients are younger than in idiopathic disease. Second, unlike in idiopathic BP mucous membranes are often involved. In addition, once the implicated drug is stopped, spontaneous resolution often occurs. $\left[{ }^{1}\right]$ Our patient first presented with blisters in the mucous membranes which subsequently spread to involve her feet and fingers and had resolution of skin lesions without any immunosuppressive therapy.

The temporal relationship between the initiation of celcoxib and capecitabine and the development of BP and subsequently the prompt resolution of bullae after drug discontinuation suggest that the BP was associated with either drug. The patient's course was complicated by mucositis. The patient had previously had severe mucositis when treated with 5-FU. Since capecitabine is metabolized to 5-FU preferentially at tumor site, it offers the potential of less gastrointestinal toxicity. $\left.{ }_{3}\right]$ Although rare, mucositis has been reported as a side effect of capecitabine therapy.[ $\left.{ }_{4}\right]$ In addition, given our patient's severe mucositic toxicity with both 5-FU and capecitabine, dihydropyrimidine dehydrogenase (DPD) deficiency, a pharmacogenetic syndrome resulting in severe toxicity to 5-FU and derivatives due to DPD deficiency, was considered.[5] We believe that the bullous eruption was a separate adverse event related either to the celecoxib, capecitabine, or both. Bullous pemphigoid secondary to either celecoxib or capecitabine has never been reported in the literature.

Although this is the first report of celecoxib or capecitabineinduced BP, there have been many reports of sulfonamideinduced BP, especially furosemide.[6] Of interest, celcoxib has a sulfonamide component in its structure. Sulfonamide hypersensitivity reactions are theorized to occur as a result of covalent linkages between macromolecules and reactive sulfonamide intermediaries creating a haptenated structure. These structures are viewed as foreign leading to an immune response.[7] This is also the theorized pathogenesis of druginduced BP: the inducing drug may act as a hapten altering the antigenicity of the lamina lucida and eliciting the formation of autoantibodies. $\left[{ }^{1}\right]$ Interestingly, our patient had previously had an allergic reaction to sulfa drugs although it did not manifest as a rash.

In addition, celecoxib has been reported to cause other cutaneous adverse effects. There have been several cases of toxic epidermal necrolysis (TEN) related to celecoxib reported in the literature. $\left[{ }_{8}, 9\right]$ TEN and BP are both on the heterogenous spectrum of subepidermal blistering diseases.

Therefore, although the BP could have been due to either the celecoxib or capecitabine, the celecoxib seems the likely culprit. Even though our case was complicated by concurrent mucositis, it represents the first report of celecoxib-induced BP. It is important to differentiate between concurrent processes to prevent recurrence of drug-induced bullous BP.

\section{CORRESPONDENCE TO}

Madeleine Duvic, M.D. Deputy Chairman, Department of Dermatology Professor, Dermatology and Internal Medicine The University of Texas M.D. Anderson Cancer Center Department of Dermatology, Unit 4341515 Holcombe Boulevard Houston, TX 77030-4095 Phone: (713) 745-1113 Fax: (713) 745-3597 E-mail: mduvic@mdanderson.org

\section{References}

1. Vassileva S: Drug-Induced Pemphigoid: Bullous and 
Cicatricial. Clin Dermatol 1998;16:379-387.

2. Czechowicz RT, Reid CM, Warren LJ, Weightman W, Whitehead FJ: Bullous pemphigoid induced by cephalexin. Austral J Dermatol 2001;42:132-135.

3. Budman DR: Capecitabine. Investigational New Drugs 200;18:355-363.

4. Bell KA, Perna AG, Hsu S: Mucositis as a treatmentlimiting side effect in the use of capecitabine for the treatment of metastatic breast cancer. J Am Acad Dermatol 2001; 45:790-791.

5. Johnson MR, Diasio RB: Importance of dihydropyrimidine dehydrogenase (DPD) deficiency in patients exhibiting toxicity following tretment with 5- fluorouracil. Advan. Enzyme Regul 2001; 41:151-157

6. Baz K, Ikizoglu G, Kaya TI, Koca A: Furosemide-induced bullous pemphigoid. JEADV 2002; 16:81-82.

7. Patterson R, Bello AE, Lefkowith J: Immunologic tolerability profile of celecoxib. Clinical Therapeutics 1999; 21:2065-2079.

8. Berger P, Dwyer D, Corallo CE. Toxic epidermal necrolysis after celecoxib therapy. Pharmacotherapy 2002; 22:1193-1195.

9. Friedman B, Orlet HK, Still JM, Law E. Toxic epidermal necrolysis due to administration of celecoxib (Celebrex). South Med J 2002; 10:1213-1214. 


\section{Author Information}

Deborah Yang, B.A.

Baylor College of Medicine

Isaac Brownell, M.D.

Baylor College of Medicine

Madeleine Duvic, M.D.

Department of Dermatology, The University of Texas M.D. Anderson Cancer Center 